\title{
INTEGRAL REPRESENTATIONS OF HERMITIAN FORMS OVER LOCAL FIELDS
}

\author{
BY ARNOLD A. JOHNSON 1
}

\author{
Communicated by G. Whaples, August 18, 1965
}

The purpose of this note is to state the solution to the problem of local integral representation of hermitian forms when the characteristic is not 2. Hermitian and quadratic forms are two of the three main types of reflexive forms (cf. [1]). Hermitian theory of forms of ten follows quickly once the corresponding quadratic theory is known. However, the local integral representation theory for quadratic forms is still incomplete and finding a general solution there appears to be very difficult.

Several results concerning these questions have been obtained over rings and fields of arithmetic type. Questions of fractional equivalence and representation as well as local integral equivalence have been solved for both quadratic and hermitian forms (cf. [2], [3], [4], [7]). Partial solutions to the problem of local integral representation of quadratic forms have been obtained by O'Meara [6] and Riehm [8]. Global results on the equivalence of hermitian forms have been obtained by Shimura [9].

The solution to the question of local integral representation of hermitian forms is obtained through three cases. We are given a local field $E$ (of characteristic $\neq 2$ ) with a nontrivial involution *. Let $F$ be the fixed field of $*$. Then the three cases considered are those where the field extension $E / F$ is (1) unramified, (2) ramified with $E$ nondyadic and (3) ramified with $E$ dyadic. The solutions in the first two cases are identical and can be stated quite easily. The third case is more complicated and some added notation will be necessary. Detailed proofs of these results will appear elsewhere.

1. Notation. Let | | denote the valuation on $E$. Let $\mathcal{D}$ be the ring of integers in $E, \mathfrak{u}$ the group of units and $p$ a prime element of $E$. The norm and trace of an element of $E$ are defined as usual; set $\pi=N(p)$. It is possible to write $E=F(\sqrt{ } \theta)$ where $\theta$ has one of the following three forms:

$$
\theta=\left\{\begin{array}{l}
1+4 \delta, \\
1+\pi^{2 k+1} \delta, \\
\pi \delta,
\end{array}\right.
$$

${ }^{1}$ Research partially supported by National Science Foundation grant GP-3986. 
for some positive integer $k$ and some unit $\delta$. Depending on the above form of $\theta$, the field extension is called unramified (U), ramified unit (R-U) or ramified prime (R-P) respectively.

Let $V$ be an hermitian space, i.e. a finite dimensional vector space $V$ over $E$ supplied with an hermitian form $q$. We denote the discriminant of $V$ by $d V$. A linear mapping of one D-lattice, in an hermitian space, into another is called a representation if it preserves the hermitian form. The problem is then: given two lattices $L$ and $K$, determine necessary and sufficient conditions that $L$ be represented by $K$, i.e. that there exists a representation $\phi: L \rightarrow K$. (Henceforth, we shall use the notation $L \rightarrow K$ and $E L \rightarrow E K$ to denote integral and fractional representation respectively.) By the usual method, we may restrict ourselves to considering only regular lattices. The scale $\& L$ and norm $\mathfrak{n} L$ of a lattice $L$ are defined as usual, i.e. the $\mathfrak{D}$-ideals generated by $\{q(x, y) \mid x, y \in L\}$ and $\{q(x, x) \mid x \in L\}$ respectively. Also the volume $\mathfrak{v} L$ is defined to be the $\mathfrak{D}$-ideal generated by the discriminant of an $\mathfrak{D}$-base for $L . L$ is called a modular lattice if $(8 L)^{n}=\mathfrak{b} L$ where $n$ is the rank of $L$.

Every lattice has a Jordan splitting, i.e.

$$
L=L_{1} \perp L_{2} \perp \cdots \perp L_{t}
$$

where each $L_{i}$ is modular and $\& L_{1} \supset \& L_{2} \supset \cdots \supset \& L_{t}$. For a given Jordan splitting (2) and for all $i$, we write $L_{(i)}=0$ if $p^{i} \supset \Omega L$ and otherwise

$$
L_{(i)}=L_{1} \perp L_{2} \perp \cdots \perp L_{\mu}
$$

where $L_{1}, L_{2}, \cdots, L_{\mu}$ are all of the components of the splitting whose scales contain $p^{i} \mathfrak{D}$. For $1 \leqq j \leqq t$, we write

$$
L_{[j]}=L_{1} \perp L_{2} \perp \cdots \perp L_{j} .
$$

2. The unramified and ramified nondyadic cases. Here we assume that the field extension $E / F$ is either unramified, or ramified with $E$ nondyadic.

Theorem 1. Let $L=L_{1} \perp L_{2} \perp \cdots \perp L_{t}$ and $K=K_{1} \perp K_{2} \perp \cdots \perp K_{8}$ be two Jordan splittings. Then $L \rightarrow K$ if and only if $E L_{(i)} \rightarrow E K_{(i)}$ for all $i$.

This reduces the problem of representation of lattices to one of representation of spaces, which has already been solved (see [4] and [7]). Note that this solution is identical to that of quadratic forms in the nondyadic case, [6].

3. The ramified dyadic case. We now assume that $E / F$ is a rami- 
fied extension and $E$ is dyadic. The solution for two modular lattices can be easily stated as follows.

THEOREM 2. Let $L$ and $K$ be two modular lattices with $8 L \subseteq \& K$, $\mathfrak{n} L \subseteq \mathfrak{n} K$, and $E L \rightarrow E K$. Then $L \rightarrow K$ if and only if $\operatorname{dim} K>\operatorname{dim} L$ or $(\xi L)^{2}(\mathfrak{n} L)^{-1} \subseteq(8 K)^{2}(\mathfrak{n} K)^{-1}$.

For any unit $\epsilon \in \mathfrak{U} \cap F$, we consider representations of $\epsilon$ in the form, $\epsilon=f f^{*}+\delta$ and write

$$
\delta(\epsilon)=\cap \delta \mathfrak{D}
$$

the intersection being taken over all such representations. $\delta(\epsilon)$ is called the normic defect of $\epsilon$.

LEMma 1. For every $\epsilon \in \mathfrak{U} \cap F$ we have

(1) $\mathfrak{D}(\epsilon)=0$ or $4 \mathfrak{D}$ in the (R-P) case,

(2) $\mathfrak{b}(\epsilon)=0$ or $4 \pi^{-2 k-1} \mathfrak{D}$ in the (R-U) case,

where $k$ is that integer appearing in (1). Moreover, there is a unit that is not a norm.

Because of this lemma, we can fix a unit $\Delta_{0}=1+\rho_{0}$ that is not a norm; where $\rho_{0}$ satisfies

$$
\rho_{0}= \begin{cases}4 \delta & \text { in the (R-P) case, } \\ 4 \pi^{-2 k-1} \delta & \text { in the (R-U) case }\end{cases}
$$

for some $\delta \in \mathfrak{U} \cap F$.

To every lattice $L$ and every $\mathfrak{D}$-ideal $\mathfrak{A}$, we can associate a sublattice

$$
L^{A}=\{x \in L \mid q(x, L) \subseteq \mathfrak{A}\}
$$

Given a Jordan splitting (2), we define the $j$ th fundamental scale and norm of $L$ by $\varepsilon_{j} L=\mathfrak{g}\left(L^{8 L \jmath}\right)$ and $\mathfrak{n}_{j} L=\mathfrak{n}\left(L^{8 L j}\right)$ for $1 \leqq j \leqq t$. If $K=K_{1} \perp K_{2} \perp \cdots \perp K_{s}$ is another Jordan splitting with $\& K \supseteq \& L$, we define a map $\lambda:\{1,2, \cdots, t\} \rightarrow\{1,2, \cdots, s\}$ by setting

$$
\lambda j= \begin{cases}s & \text { if } \& K_{s} \supseteq \& L_{j}, \\ \mu & \text { where } \& K_{\mu} \supseteq \& L_{j} \supset \mathfrak{B} K_{\mu+1} .\end{cases}
$$

With this added notation, we can now state the main result.

Theorem 3. Let $L=L_{1} \perp L_{2} \perp \cdots \perp L_{t}$ and $K=K_{1} \perp K_{2} \perp \cdots \perp K_{8}$ be two Jordan splittings; and suppose $\mathfrak{n} L \subseteq \mathfrak{n} K$ and $B L \subseteq \& K$. Then $L \rightarrow K$ if and only if:

(1) $\operatorname{dim} L_{[j]} \leqq \operatorname{dim} K_{[\lambda j]}$ for $1 \leqq j \leqq t$,

(2) when $\operatorname{dim} L_{[j]}=\operatorname{dim} K_{\left[\lambda_{j}\right]}$, we have 
(2a) $\mathfrak{n}_{j+1} L \subseteq \mathfrak{n}_{\left(\lambda_{j}\right)+1} K+\left(\mathbb{B}_{\left(\lambda_{j}\right)+1} K\right)^{2}\left(\mathbb{B}_{j} L\right)^{-2} \mathfrak{n}_{j} L$,

(2b) $d\left(E L_{[j]}\right)=d\left(E K_{[\lambda j]}\right)$ or $\mathfrak{n}_{(\lambda j)+1} K \supseteq \rho_{0}\left(\mathbb{z}_{\lambda j} K\right)^{2}\left(\mathfrak{n}_{\lambda j} K\right)^{-1}$,

(2c) $\left(\mathfrak{g}_{j} L\right)^{2}\left(\mathfrak{n}_{j} L\right)^{-1} \subseteq\left(\mathfrak{g}_{\lambda_{j}} K\right)^{2}\left(\mathfrak{n}_{\lambda j} K\right)^{-1}$ or $\mathfrak{n}_{\left(\lambda_{j}\right)+1} K \supseteq\left(\mathbb{B}_{j} L\right)^{2}\left(\mathfrak{g}_{\lambda j} K\right)^{-2} \mathfrak{n}_{\lambda j} K$.

A cancellation theorem, of Witt type for hyperbolic planes under representation, can be obtained from Theorem 3 .

THEOREM 4. Let $L$ and $K$ be two lattices and $H$ be a hyperbolic plane. Then $L \rightarrow K$ if and only if $L \perp H \rightarrow K \perp H$.

\section{BIBLIOGRAPHY}

1. Jean Dieudonné, La géometrie des groupes classiques, 2nd ed., Springer, Berlin, 1963.

2. H. Hasse, Äquivalenz quadratische Formen in einem beliebigen algebraischen Zahlkörper, J. Reine Angew. Math. 153 (1924), 158-162.

3. Ronald Jacobowitz, Hermitian forms over local fields, Amer. J. Math. 86 (1962), 441-465.

4. N. Jacobson, $A$ note on hermitian forms, Bull. Amer. Math. Soc. 46 (1940), 264-268.

5. O. T. O'Meara, Integral equivalence of quadratic forms in ramified local fields, Amer. J. Math. 79 (1957), 157-186.

6. - The integral representations of quadratic forms over local fields, Amer. J. Math. 80 (1958), 843-878.

7. - Introduction to quadratic forms, Springer, Berlin, 1963.

8. Carl Riehm, On integral representations of quadratic forms over local fields, Amer. J. Math. 86 (1964), 25-62.

9. Goro Shimura, Arithmetic of unitary groups, Ann. of Math. 79 (1964), 369-409.

UNIVERSITY OF Notre DAME 